\title{
Exercise and Sodium Butyrate Transform a Subthreshold Learning Event into Long-Term Memory via a Brain-Derived Neurotrophic factor-Dependent Mechanism
}

\author{
Karlie A Intlekofer', Nicole C Berchtold*,', Melissa Malvaez², Anthony J Carlos', Susan C McQuown², \\ Michael J Cunningham ${ }^{3}$, Marcelo A Wood ${ }^{2}$ and Carl W Cotman' \\ 'Institute for Memory Impairments and Neurological Disorders, University of California Irvine, Irvine, CA, USA; '² Department of Neurobiology and \\ Behavior, Center for the Neurobiology of Learning and Memory, University of California, Irvine, CA, USA; ${ }^{3}$ University of Vermont College of \\ Medicine, Burlington, VT, USA
}

\begin{abstract}
We demonstrate that exercise enables hippocampal-dependent learning in conditions that are normally subthreshold for encoding and memory formation, and depends on hippocampal induction of brain-derived neurotrophic factor (BDNF) as a key mechanism. Using a weak training paradigm in an object location memory (OLM) task, we show that sedentary mice are unable to discriminate $24 \mathrm{~h}$ later between familiar and novel object locations. In contrast, 3 weeks of prior voluntary exercise enables strong discrimination in the spatial memory task. Cognitive benefits of exercise match those attained with post-training sodium butyrate ( $\mathrm{NaB}$ ), a histone deacetylase (HDAC) inhibitor previously shown to enable subthreshold learning. We demonstrate that the enabling effects of exercise and $\mathrm{NaB}$ on subthreshold OLM learning are dependent on hippocampal BDNF upregulation, and are blocked by hippocampal infusion of BDNF short-interfering RNA. Exercise and $\mathrm{NaB}$ increased bdnf transcripts I and IV, and the increases were associated with BDNF promoter acetylation on $\mathrm{H} 4 \mathrm{~K} 8$ but not H4K I2. These data provide support for the concept that exercise engages epigenetic control mechanisms and serves as a natural stimulus that operates in part like $\mathrm{NaB}$ and potentially other HDAC inhibitors, placing the brain into a state of readiness for plasticity.

Neuropsychopharmacology (2013) 38, 2027-2034; doi:I0.1038/npp.20I3.104; published online I5 May 20I3
\end{abstract}

Keywords: object location memory; hippocampus; siRNA; subthreshold; plasticity

\section{INTRODUCTION}

Exercise is well established to improve brain health and function in both humans and other animals (Cotman et al, 2007; Middleton et al, 2010; Ahlskog et al, 2011; Voss et al, 2011). Animal studies reveal improved performance in numerous hippocampal-dependent tasks following several days to weeks of exercise participation (Cotman and Berchtold, 2002; Cotman et al, 2007; Bekinschtein et al, 2011). An idea that has not been tested is that exercise may enable hippocampal-dependent learning in conditions that are normally subthreshold for encoding and memory formation. In particular, a weak training paradigm that is normally insufficient (subthreshold) for encoding may become sufficient for long-term memory formation in an animal that has engaged in physical activity.

\footnotetext{
* Correspondence: Dr NC Berchtold, Institute for Memory Impairments and Neurological Disorders, University of California Irvine, 1206 Gillespie Building, Irvine, CA 92697-4260, USA, Tel: + I 949824 607I, Fax: + I 949824 207I, E-mail: nberchto@uci.edu

Received 5 March 2013; revised 12 April 2013; accepted 14 April 2013; accepted article preview online 24 April 2013
}

Exercise induces multiple mechanisms in the hippocampus that support the possibility that exercise may improve the acquisition and consolidation of normally subthreshold training. For example, exercise increases hippocampal expression of several plasticity-related growth factors including brain-derived neurotrophic factor (BDNF; Neeper et al, 1996; Cotman and Berchtold, 2002), thought to be a central mechanism mediating beneficial effects of exercise on hippocampal-dependent cognition (Cotman et al, 2007). BDNF, which is essential for certain forms of learning and memory, enhances synaptic plasticity by triggering functional and structural changes in neurons and synapses (Lu et al, 2008; Gottmann et al, 2009), such as facilitating the induction of long-term potentiation (LTP) (Rex et al, 2007), a synaptic correlate of learning and memory. Previously we have suggested that BDNF induction with exercise serves to place the brain into a state of readiness for plasticity (Cotman et al, 2007), an idea we test in this study using a subthreshold learning paradigm.

The induction of BDNF with exercise may involve epigenetic mechanisms, by which dynamic and reversible changes to chromatin structure modify the accessibility of a gene to the molecular machinery regulating transcription. One such epigenetic mechanism involves regulation of a 
gene's acetylation state by histone acetyltransferases (HATs), which increase acetylation and generally facilitate transcription, and the opposing action of histone deacetylases (HDACs), which reduce acetylation and generally silence transcription (Kouzarides, 2007). Emerging evidence demonstrates that histone acetylation regulates BDNF transcription, and that BDNF acetylation is associated with synaptic plasticity. For example, interventions that promote acetylation (eg, HDAC inhibitors such as sodium butyrate $(\mathrm{NaB})$ and valproic acid) increase BDNF expression during memory processes in vivo (Bredy et al, 2007; Zeng et al, 2011), and induce BDNF expression associated with increased quantal neurotransmitter release and dendritic spine density in hippocampal slices (Calfa et al, 2012). This literature, taken together with the role of BDNF in orchestrating cognitive benefits of exercise, suggests that exercise may alter acetylation patterns of BDNF in the hippocampus to regulate BDNF availability. Although data is emerging that exercise causes epigenetic change in the brain (Gomez-Pinilla et al, 2011; Abel and Rissman, 2012), the specific BDNF promoter acetylation sites that may underlie exercise-induced improvements to hippocampal function are not yet clear.

In this study, we address several hypotheses. First, we investigate the possibility that exercise enables hippocampal-dependent learning in conditions that are normally subthreshold for memory formation in sedentary animals. Second, we evaluate whether exercise has similar efficacy for cognitive enhancement as $\mathrm{NaB}$, an $\mathrm{HDAC}$ inhibitor that has been shown to enable subthreshold learning (Stefanko et al, 2009; McQuown et al, 2011). Third, we investigate the patterns of $b d n f$ transcript induction in the hippocampus following exercise and $\mathrm{NaB}$, and assess the relationship of $b d n f$ transcript induction and $b d n f$ promoter acetylation. Finally, we investigate whether hippocampal induction of $b d n f$ transcription is necessary for exercise and $\mathrm{NaB}$ to enable subthreshold learning, using short-interfering RNA (siRNA) against $b d n f$, thereby assessing the possibility that exercise and $\mathrm{NaB}$ facilitate encoding of a hippocampaldependent task by a common mechanism. To address these hypotheses, we use a location-dependent object recognition memory task (OLM) with a weak training paradigm that is normally insufficient to allow for short- or long-term memory formation in sedentary animals. Importantly, this subthreshold OLM paradigm can be encoded into long-term memory by treatment with $\mathrm{NaB}$ (Stefanko et al, 2009; McQuown et al, 2011). We predict that like $\mathrm{NaB}$, exercise will enable subthreshold learning, allowing us to compare BDNF transcript induction and promoter acetylation patterns between the two interventions.

\section{MATERIALS AND METHODS}

\section{Animals and General Study Design}

Male mice (C57Bl/6J, 6 weeks; Jackson Laboratory), were individually housed with food and water ad libitum, and were acclimated to the vivarium for 1 week before experimental procedures. Lights were maintained on a $12: 12$ light/dark cycle, and all behavior testing was carried out during the light phase of the cycle. Exercised animals were individually housed in cages equipped with a running wheel, and the distance run was monitored by automated a

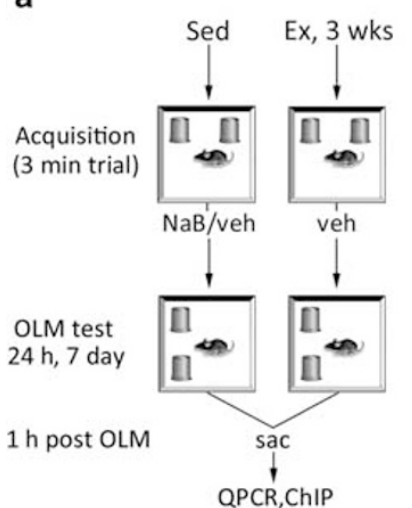

b

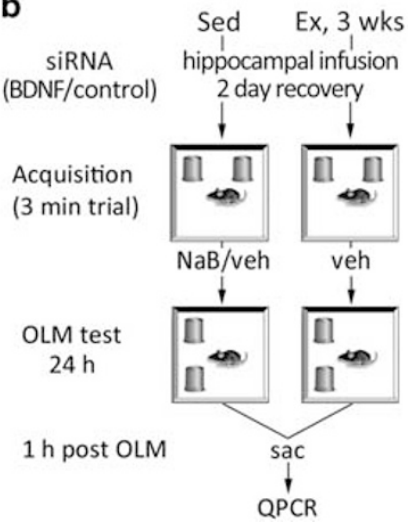

Figure I Experimental design. (a) Sedentary (Sed) or exercised (EX) mice were given a 3-min acquisition trial (as a subthreshold learning task as shown previously; Stefanko et al, 2009; McQuown et al, 20 I I), followed immediately with an i.p. injection of sodium butyrate $(\mathrm{NaB})$ or vehicle (veh), and returning all animals to clean home cages without access to running wheels. Twenty-four hours or 7 days later, a 5-min object location memory (OLM) retention test was administered where one object was moved to a novel location. Time spent exploring the identical objects was recorded and a discrimination index (DI) was calculated to determine preference for the object in the novel location. (b) To determine whether a brain-derived neurotrophic factor (BDNF)-dependent mechanism was involved in the enhancement of subthreshold learning, short-interfering RNA (siRNA) against bdnf, or control siRNA, were infused to the hippocampus 2 days before OLM acquisition. Mice were given a 3-min acquisition trial, followed $24 \mathrm{~h}$ later by a 5 -min OLM retention test. Animals were killed I h after the OLM test, and hippocampi were removed for molecular assays.

counters interfaced with computer software. Subthreshold OLM training and testing procedures were performed as described previously (Stefanko et al, 2009). Briefly, mice were handled for $2 \mathrm{~min}$ per day for 5 days, followed by habituation to the experimental apparatus (white rectangular open field measuring $30 \times 23 \times 21.5 \mathrm{~cm}$ ) for 5 min per day for 4 consecutive days before training. Mice were given a 3-min acquisition trial that has been shown previously to be subthreshold for learning in sedentary animals (Stefanko et al, 2009; McQuown et al, 2011), followed immediately with an i.p. injection of $\mathrm{NaB}$ or vehicle, and all animals were placed in clean home cages without access to running wheels. Twenty-four hours and 7 days later, a 5-min retention test was administered, where one object was moved to a novel location (Figure 1a). To determine whether a $b d n f-$ dependent mechanism was involved in the enhancement of subthreshold learning, SMART pool siRNA against BDNF (Dharmacon; Thermo Fisher Scientific, Lafayette, CO) were infused to the hippocampus 2 days before OLM acquisition, using methods described previously (McQuown et al, 2011; Figure 1b). Animals were killed $1 \mathrm{~h}$ after OLM testing and hippocampi were removed, rapidly frozen on dry ice, and stored at $-80^{\circ} \mathrm{C}$ until processing for mRNA and acetylation assays.

\section{Group Sizes}

Three treatment groups ( $n=8$ per group) were tested for each of the 24-h and 7-day timepoints: (1) sedentary animals treated with vehicle (Sed/Veh), (2) sedentary animals treated with $\mathrm{NaB}(\mathrm{Sed} / \mathrm{NaB})$, and exercised animals 
with 21-day running wheel access, receiving vehicle (Ex/Veh). Tissue limitations required that acetylation assessment was conducted with half of the samples each for $\mathrm{H} 4 \mathrm{~K} 8$ and $\mathrm{H} 4 \mathrm{~K} 12$ acetylation assays (eg, $n=4$ per group). Eight mice that were not exposed to handling or injections were used as negative controls in chromatin immunoprecipitation (ChIP) assays to establish baseline acetylation levels. For the exercise arm of the siRNA study, three treatment groups were used $(n=12$ per group): Sed/Control siRNA, Ex/Control siRNA, and Ex/bdnf siRNA. For the $\mathrm{NaB}$ arm of the siRNA study, the two treatment groups ( $n=10$ per group) were: (1) Sed/NaB/Control siRNA and (2) Sed/NaB/bdnf siRNA. For detailed methods see the Supplementary Information available at the Neuropsychopharmacology website.

\section{Quantitative PCR and Acetylation Assay}

Gene expression for total $b d n f$ and specific $b d n f$ transcripts was assessed by quantitative PCR following reverse transcription (RT-qPCR). Total $b d n f$ primers were designed to the common $3^{\prime}$ coding exon. Primers for $b d n f$ transcripts I, IV, and VI correspond to each unique exon sequence (Aid et al 2007; Supplementary Figure S1). RT-qPCR primer sets (Supplementary Table S1) were designed using the Roche Universal Probe Library Assay Design Center and obtained from Integrated DNA Technologies (Coralville, IA). RT-qPCR reactions were run in a Stratagene MX3005P thermocycler at $95{ }^{\circ} \mathrm{C}$ for $3 \mathrm{~min}$, followed by 45 cycles of $95^{\circ} \mathrm{C}$ for $10 \mathrm{~s}$, and $58^{\circ} \mathrm{C}$ for $15 \mathrm{~s}$. Each RT-qPCR run included all samples run in triplicate and a standard curve. Data were analyzed by the $2^{-\Delta \Delta \mathrm{Ct}}$ method and expressed as fold change over control after normalizing with input samples, as described previously (Sahar et al, 2007). ChIP assay was followed by RT-qPCR for specific BDNF promoters to assess histone acetylation (H4K8 and $\mathrm{H} 4 \mathrm{~K} 12$ ) at $b d n f$ promoter regions (see Supplementary Table 2 for RT-qPCR primer sequences used in conjunction with ChIP). Chromatin extraction from hippocampal tissue was performed using the 'EZ-Magna ChIP-A Chromatin Immunoprecipitation Kit' (Millipore, Billerica, MA), following the manufacturer's protocol. The following antibodies were used for immunoprecipitation: anti-acetyl histone $\mathrm{H} 4$ lysine 8 (H4K8Ac; Millipore), anti-acetyl histone H4 lysine 12 (H4K12Ac; Abcam, Cambridge, MA), anti-acetylated H3 (positive control; Millipore), and non-immune rabbit IgG (negative control; Millipore).

\section{Statistics}

Treatment effects were detected using one-way analysis of variance, followed by post hoc Bonferroni's $t$-tests to delineate between-group differences.

For additional details, see Supplementary Materials and Methods at the Neuropsychopharmacology website.

\section{RESULTS}

Exercise Enables Learning in a Training Paradigm that is Normally Subthreshold for Long-Term Memory

Previously it has been demonstrated that a 3-min training session in a location-dependent OLM task is normally
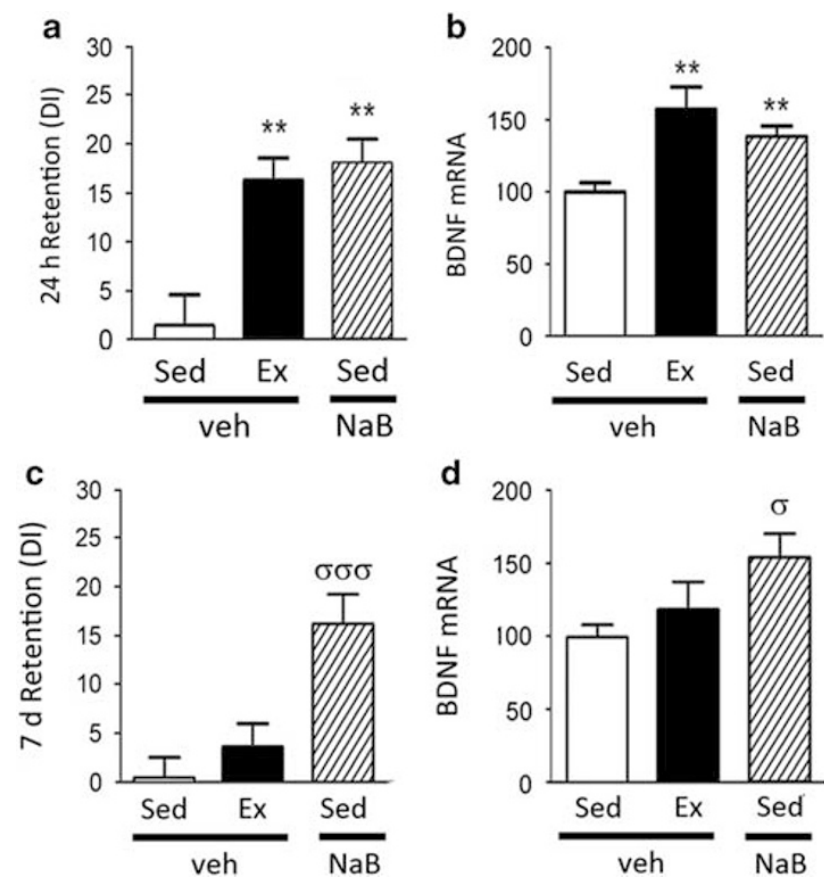

Figure 2 In a subthreshold object location memory (OLM) paradigm, exercise (Ex) improves learning equivalent to sedentary (Sed) mice injected with post-acquisition sodium butyrate ( $\mathrm{NaB})$, a histone deacetylase (HDAC) inhibitor. (a) Twenty-four hours after a 3-min acquisition trial, vehicle-treated Sed animals do not show preference for the novel location over the familiar location, as measured by the discrimination index ratio (DI). Following 3 weeks voluntary exercise, Ex animals show marked preference for the novel location to a similar extent as post-acquisition injection of $\mathrm{NaB}$. (b) Quantitative reverse transcription PCR (RT-qPCR) revealed that hippocampal brain-derived neurotrophic factor (bdnf) mRNA levels are increased by exercise and $\mathrm{NaB}$, in parallel with the cognitive enhancement. (c) Effects of $\mathrm{NaB}$ on cognition persist 7 days after the acquisition trial, whereas the exercise effects on spatial memory in the subthreshold paradigm eventually decay after daily physical activity ceases. (d) RT-qPCR revealed that bdnf mRNA expression patterns at the 7-day timepoint paralleled the cognitive data across the treatment groups, remaining elevated after $\mathrm{NaB}$ treatment but not with exercise. Data are expressed as means \pm SEM. For the 24-h timepoint: $* * *<0.01$ vs sedentary. For the 7-day timepoint: ${ }^{\sigma_{p}}<0.05 ;{ }^{\sigma \sigma \sigma_{p}}<0.00$ I vs sedentary.

insufficient, or subthreshold, to allow for short- or longterm memory formation in sedentary animals (Stefanko et al, 2009; McQuown et al, 2011). We investigated the possibility that exercise may enable learning in subthreshold conditions. Mice were provided free access to running wheels for 3 weeks, followed by subthreshold OLM training ( $3 \mathrm{~min})$, and $24 \mathrm{~h}$ later, assessment of long-term memory. In parallel, we compare the efficacy of 3 weeks exercise with post-training $\mathrm{NaB}$ treatment, an HDAC inhibitor previously been shown to enable consolidation of the subthreshold event (see Figure 1a for Experimental Design).

The 24-h retention test revealed a significant effect of treatment on OLM performance $\left(\mathrm{F}_{(2,21)}=10.14, p<0.001\right)$. Confirming that the training paradigm is normally subthreshold for learning, sedentary mice were unable to discriminate between familiar and novel object locations in the 24 -h retention test (Figure 2a). In contrast, exercised animals showed significant discrimination and long-term memory, with exercise similarly effective as post-training 
injection of $\mathrm{NaB}$ (Figure 2a). These data reveal that exercise enables learning in conditions that are normally subthreshold for memory formation, and that exercise facilitates encoding to a similar extent as $\mathrm{NaB}$, a memory-enhancing drug.

\section{Exercise- and NaB-induced Gains in OLM Performance are Associated with $b d n f$ Transcription}

We next investigated the possibility that a common molecular mechanism may be engaged by exercise and $\mathrm{NaB}$ that enables encoding of normally subthreshold learning conditions. Specifically, we evaluated whether exercise and $\mathrm{NaB}$ induce hippocampal transcription of $b d n f$, a plasticity gene central to hippocampal-dependent learning. Hippocampal $b d n f$ expression was evaluated $1 \mathrm{~h}$ after the 24-hour OLM retention test by RT-qPCR. There was a significant effect of treatment on $b d n f$ gene expression $\left(\mathrm{F}_{(2,21)}=11.99\right.$, $p<0.001$ ), with both exercise and $\mathrm{NaB}$ significantly increasing bdnf mRNA over sedentary levels (1.60-fold and 1.37-fold, respectively; Figure 2b).

As transcription of $b d n f$ is regulated by several exons, of which $b d n f$ I, IV, and VI are the most highly expressed transcripts in the hippocampus, expression levels of $b d n f \mathrm{I}$, IV, and VI transcripts were also evaluated. We found a significant effect of treatment on $b d n f \mathrm{I}\left(\mathrm{F}_{(2,21)}=4.80\right.$, $p<0.05)$ and $b d n f$ IV $\left(\mathrm{F}_{(2,21)}=4.02, p<0.05\right)$, but not $b d n f$ VI. Post hoc tests revealed that exercise and $\mathrm{NaB}$ treatments induced $b d n f$ I (Supplementary Figure S2A) and $b d n f$ IV (Supplementary Figure S2B), whereas neither treatment increased $b d n f$ VI gene expression (Supplementary Figure $\mathrm{S} 2 \mathrm{C})$. These data indicate that hippocampal induction of $b d n f$ mRNA (specifically $b d n f$ I and $b d n f$ IV) is associated with the successful encoding and consolidation, following exercise or $\mathrm{NaB}$, of a normally subthreshold task.

\section{Behavioral and Molecular Effects of $\mathrm{NaB}$ (but not Exercise) Remain Present in a 7-Day Retention Test}

It has previously been demonstrated that post-training $\mathrm{NaB}$ leads to long-term memory formation for novel objects that persists at least 7 days, lasting beyond the point at which normal memory fails (Stefanko et al, 2009). However, it is unknown whether $\mathrm{NaB}$ induces a similar persistence of memory for the hippocampal-dependent OLM task, and the relationship to BDNF has not been explored. Here, we determine whether the behavioral and molecular effects of $\mathrm{NaB}$ and exercise are long lasting, by evaluating OLM and $b d n f$ expression 7 days after the acquisition trial (see Figure 1a for Experimental Design).

OLM retention testing at 7 days after the acquisition trial revealed persistent long-term memory with $\mathrm{NaB}$ treatment but not exercise (Figure 2c), suggesting that the effects of exercise on spatial memory in the subthreshold paradigm eventually decay after daily physical activity ceases. Interestingly, the pattern of $b d n f$ mRNA expression across treatment groups paralleled the cognitive data. Specifically, memory testing 7 days after the acquisition trial revealed that exercised animals had no enduring cognitive enhancement (Figure 2c), and no significant elevation of total $b d n f$ mRNA (Figure 2d) or any bdnf transcript examined (Supplementary Figure S2 D-F). In contrast, animals treated with post-acquisition $\mathrm{NaB}$ showed persistent long-term memory (Figure 2c), along with elevated levels of total $b d n f$ (Figure $2 \mathrm{~d}$ ) at the 7 day timepoint. The elevation of total $b d n f$ was associated with increased levels of $b d n f$ IV (Supplementary Figure S2E) but not $b d n f$ I (Supplementary Figure S2D) or $b d n f$ VI (Supplementary Figure S2F). These findings indicate that exercise effects on hippocampal function and $b d n f$ gene expression patterns are reversible, whereas effects of HDAC inhibition appear to be relatively stable over time.

\section{Exercise and $\mathrm{NaB}$ Hyperacetylate H4K8-Associated $b d n f$ I and $b d n f$ IV Promoters}

As $\mathrm{NaB}$ is an HDAC inhibitor, we next investigated whether the increased transcription of $b d n f$ and its exons by $\mathrm{NaB}$ is associated with specific promoter acetylation. In parallel, we investigated whether exercise increases $b d n f$ promoter acetylation, and compared the patterns of $b d n f$ promoter acetylation associated with the $\mathrm{NaB}$ and exercise treatments. We specifically examined acetylation of H4K8Ac and H4K12Ac of bdnf promoters I, IV, and VI, based on literature that acetylation of $\mathrm{H} 4 \mathrm{~K} 8$ and $\mathrm{H} 4 \mathrm{~K} 12$ are important in memory consolidation processes (Peleg et al, 2010; McQuown et al, 2011).

A significant effect of treatment was found for $\mathrm{H} 4 \mathrm{~K} 8 \mathrm{Ac}$ at $b d n f \mathrm{I}\left(\mathrm{F}_{(3,12)}=6.98, p<0.05\right)$ and $b d n f \operatorname{IV}\left(\mathrm{F}_{(3,12)}=5.624\right.$, $p<0.05$ ) but not bdnf VI (Figure 3). Post hoc tests revealed that exercise and $\mathrm{NaB}$ each increased $\mathrm{H} 4 \mathrm{~K} 8 \mathrm{Ac}$ at $b d n f \mathrm{I}$ $(p<0.05$; Figure 3a) and $b d n f$ IV $(p<0.05$; Figure $3 b)$ with no change in $b d n f$ VI (Figure $3 c$ ), paralleling the respective mRNA patterns for the related transcripts (Supplementary Figure S2 A-C). These results suggest that increased $b d n f$ mRNA levels with exercise and $\mathrm{NaB}$ are likely regulated through H4K8Ac of BDNF. At the H4K12 acetylation site, there was a significant effect of treatment for $b d n f$ I $\left(\mathrm{F}_{(3,12)}=4.476, p<0.05\right)$, bdnf IV $\left(\mathrm{F}_{(3,12)}=5.593, p<0.05\right)$ and $b d n f$ VI promoters $\left(\mathrm{F}_{(3,12)}=3.83, p<0.05\right)$; however, post hoc tests revealed no significant increase in H4K12Ac levels in the exercise- or NaB-treated groups relative to the sedentary vehicle group at any $b d n f$ promoter (Figure $3 \mathrm{~d}-\mathrm{f}$ ). Interestingly, OLM testing on its own had a small effect on acetylation patterns relative to baseline acetylation levels (eg, sedentary-veh $v s$ baseline), upregulating promoter H4K8Ac for bdnf I (Figure 3a) and IV (Figure 3b) as well as $\mathrm{H} 4 \mathrm{~K} 12 \mathrm{Ac}$ of $b d n f$ IV (Figure 3e), suggesting that these histone modifications are highly sensitive to environmental stimulation. Finally, although acetylation of $\mathrm{H} 4 \mathrm{~K} 12$ has been shown to be key for normal memory consolidation in the aged mouse brain (Peleg et al, 2010), our results suggest that $\mathrm{H} 4 \mathrm{~K} 12$ acetylation is not involved in benefits of exercise and $\mathrm{NaB}$ on OLM learning in young mice.

Taken together, these data demonstrate that exercise, like $\mathrm{NaB}$, affects chromatin modification on the BDNF gene. Exercise and $\mathrm{NaB}$ increase acetylation at $\mathrm{H} 4 \mathrm{~K} 8$ but not H4K12, revealing highly specific and parallel epigenetic effects of exercise and $\mathrm{NaB}$ on $b d n f$ promoters. Importantly, these results indicate that exercise may permit greater access of transcriptional machinery by establishing a more accessible chromatin state, similar to the mechanisms of $\mathrm{NaB}$. 

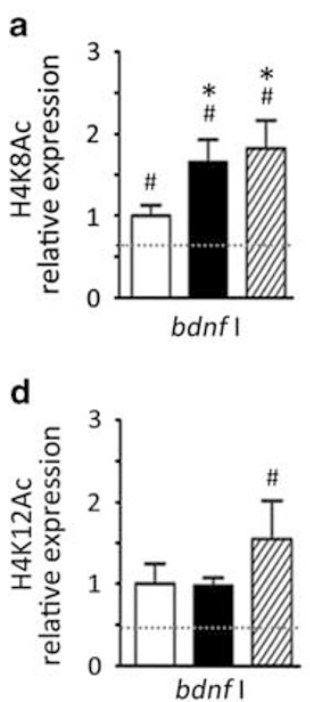

b

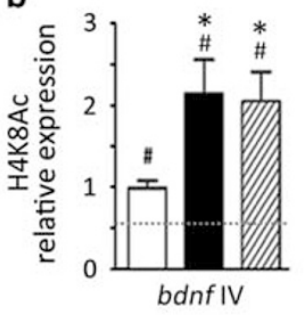

e

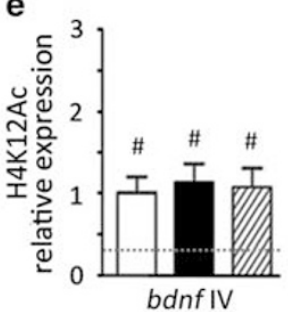

C

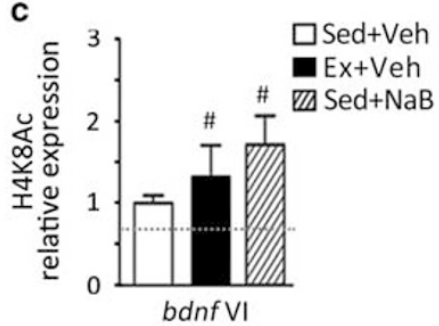

f

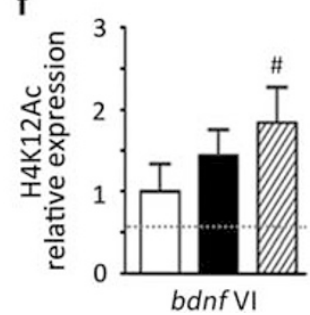

Figure 3 Exercise (Ex) and sodium butyrate $(\mathrm{NaB})$ increase acetylation of histone $\mathrm{H} 4$, specifically acetylation at $\mathrm{H} 4 \mathrm{~K} 8$ of promoters for (a) brain-derived neurotrophic factor (bdnf) I and (b) bdnf IV, but not (c) bdnf VI. Acetylation at H4KI2 was not significantly increased by Ex or NaB at either promoters (d) bdnf I, (e) bdnf IV, or (f) bdnf VI. Comparing sedentary (Sed) animals with or without object location memory (OLM) testing, behavioral testing had a small effect on H4K8 acetylation on bdnf promoters I and IV, and H4KI2 acetylation in bdnf promoter IV. Data are expressed as means \pm SEM, * $p<0.05$ vs Sed + Veh (OLM tested), ${ }^{\#} p<0.05$, vs baseline acetylation level in sedentary unhandled animals (indicated by dotted line).
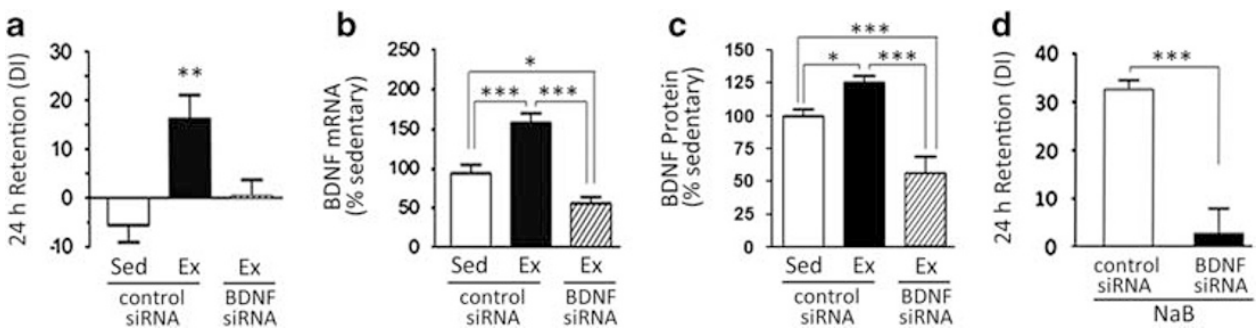

Figure 4 Preventing increased brain-derived neurotrophic factor (bdnf) mRNA expression with intrahippocampal short-interfering RNA (siRNA) blocks the cognitive benefits of exercise $(\mathrm{Ex})$ and sodium butyrate $(\mathrm{NaB})$ in a subthreshold object location memory (OLM) learning task. (a) Exercise-dependent improvements in the subthreshold OLM paradigm were abolished by intrahippocampal infusion of bdnf siRNA, but not by control siRNA. Intrahippocampal bdnf siRNA blocked induction of (b) bdnf mRNA and (c) BDNF protein expression by exercise and decreased levels of mRNA and protein below baseline. (d) Cognitive improvements in the subthreshold OLM paradigm with $\mathrm{NaB}$ treatment were blocked by intrahippocampal infusion of bdnf siRNA, but not control siRNA. Data are expressed as means \pm SEM. ${ }^{*} p<0.05$; ** $p<0.01$; ***** $p<0.001$.

\section{BDNF Induction is Required for Cognitive Enhancement by Exercise and $\mathrm{NaB}$}

Finally, we investigated whether induction of hippocampal BDNF transcription is necessary for exercise and $\mathrm{NaB}$ to transform a subthreshold learning event into robust longterm memory. siRNA targeting BDNF was infused bilaterally into the hippocampus $48 \mathrm{~h}$ before the subthreshold OLM task to selectively block elevation of hippocampal $b d n f$ mRNA by exercise or $\mathrm{NaB}$, and memory was assessed $24 \mathrm{~h}$ after the acquisition trial.

There was a significant effect of treatment for exercise and siRNA on OLM $\left(\mathrm{F}_{(2,29)}=4.80, p<0.01\right)$. Consistent with our previous findings, exercised animals treated with control siRNA showed significant discrimination and learning at the 24-h memory test after subthreshold training (Figure 4a). In contrast, BDNF siRNA prevented the cognitive enhancement by exercise, such that the exercise/ BDNF siRNA group showed no significant preference for the object in the novel location, similar to sedentary controls (Figure 4a). In parallel with the effect on cognitive performance, BDNF siRNA prevented the induction of $b d n f$ gene expression (Figure $4 \mathrm{~b}$ ) and BDNF protein (Figure 4c) in response to exercise, while in the presence of control siRNA, exercise increased bdnf gene expression 1.6-fold over sedentary/control siRNA levels (Figure $4 \mathrm{~b}$ ). These data demonstrate that induction of $b d n f$ mRNA is required for exercise to transform a normally subthreshold learning event into long-term memory.

Similarly, our data reveal that induction of $b d n f$ mRNA is necessary for the cognitive facilitation by $\mathrm{NaB}$. Specifically, whereas NaB-treated mice infused with control siRNA exhibited significant preference for the object in the novel location, NaB-treated mice infused with BDNF siRNA exhibited no preference (Figure 4d), demonstrating that $\mathrm{BDNF}$ is necessary for HDAC inhibition via $\mathrm{NaB}$ to transform a subthreshold learning event into long-term memory. Interestingly, BDNF siRNA reduced levels of hippocampal 
$b d n f$ mRNA and protein below baseline expression to $61 \%$ of sedentary controls (Figure $4 \mathrm{~b}$ and c), predominantly because of reductions below baseline of $b d n f$ transcript IV (Supplementary Figure S3B) and bdnf VI (Supplementary Figure S3C), whereas no effect on $b d n f I$ was observed (Supplementary Figure S3A). The drop below baseline in the presence of BDNF siRNA is consistent with the fact that $b d n f$ mRNA undergoes turnover in the hippocampus, even in sedentary animals.

Taken together, these data demonstrate that induction of $b d n f$ mRNA with exercise and $\mathrm{NaB}$ is necessary to transform a subthreshold learning event into robust long-term memory, such that exercise and $\mathrm{NaB}$ facilitate encoding in a hippocampal $b d n f$-dependent manner.

\section{DISCUSSION}

In this study, we report the novel finding that exercise enables the acquisition of subthreshold experiences, such that a hippocampal-dependent learning event that would not normally be remembered is encoded into long-term memory. Remarkably, exercise-induced improvements in the subthreshold learning paradigm were similar to those attained by treatment with $\mathrm{NaB}$ (Stefanko et al, 2009), a memory-enhancing drug. Our data demonstrate that the enabling effect of exercise and $\mathrm{NaB}$ on subthreshold OLM learning is dependent on BDNF upregulation, and is prevented by hippocampal infusion of BDNF siRNA.

The finding that exercise, like $\mathrm{NaB}$, enables the acquisition of subthreshold experiences adds to growing evidence that exercise alters the sensitivity of hippocampal neural networks and enhances learning (Cotman and Berchtold, 2007). In addition, these data provide a behavioral readout consistent with electrophysiology data that a weak LTP induction paradigm that is normally subthreshold becomes a sufficient induction stimulus for LTP after exercise (Farmer et al, 2004) or exposure to $\mathrm{NaB}$ (Vecsey et al, 2007). Finally, the demonstration that OLM is facilitated following exercise reveals that voluntary physical activity improves a non-reinforced form of hippocampal-dependent learning, complementing previous findings that exercise improves learning in negatively motivated hippocampaldependent tasks (such as the Morris water maze, which relies on an intention to escape a swimming stressor; van Praag et al, 1999; Vaynman et al, 2004; Berchtold et al, 2010; Cassilhas et al, 2012). OLM, being a non-reinforced form of learning, may more closely approximate the type of learning most prevalent in everyday life for humans, where learning accompanies daily activities in the absence of explicit instruction or positive/negative reinforcement.

Our finding that the enabling effect of exercise and $\mathrm{NaB}$ on encoding is blocked in the presence of BDNF siRNA is consistent with several lines of evidence demonstrating a key role for BDNF in various hippocampus-dependent spatial memory tasks (Ma et al, 1998; Mu et al, 1999; Mizuno et al, 2000; Gorski et al, 2003; Vaynman et al, 2004). The data underscore the requirement for hippocampal BDNF signaling in spatial memory, and support the concept that physical activity elevates BDNF to levels that enhance hippocampal function. In addition, the finding that exercise increases hippocampal levels of $b d n f$ I and IV (but not VI) builds on previous evidence that physical activity selectively increases expression of these transcripts (Oliff et al, 1998; Russo-Neustadt et al, 2000). Finally, our data reveal that $\mathrm{NaB}$ targets the same $b d n f$ transcripts as exercise, upregulating $b d n f$ I and IV, but not $b d n f$ VI. These data suggest that hippocampal expression of $b d n f$ I and IV may be triggered by common signaling pathways as part of the adaptive response to exercise or $\mathrm{NaB}$, and serve to facilitate learning.

In parallel with assessing performance benefits of exercise and $\mathrm{NaB}$ at $24 \mathrm{~h}$ after OLM training, a timepoint that informs whether learning was encoded and transferred into long-term memory, we extended our analysis to 7 days after the acquisition trial to assess the stability of the memory enhancement. The enhancing effects of $\mathrm{NaB}$ on memory endured at the 7-day timepoint, consistent with previous findings (Stefanko et al, 2009; McQuown et al, 2011), and the effects on spatial memory were paralleled by sustained increases in total $b d n f$ and $b d n f$ transcripts I and IV. In contrast, the enhancement of OLM by exercise was not maintained after 7 days, and the loss of cognitive enhancement was paralleled by return of $b d n f$ mRNA to baseline levels. These findings indicate that the effects of exercise on hippocampal function and $b d n f$ gene expression patterns are reversible, while effects of HDAC inhibition appear to be relatively stable over time. It is important to note that a critical aspect of plasticity is cognitive flexibility, to allow for the rapid adaptation to changing rules and for previous associations to be updated.

Finally, the data reveal that exercise and $\mathrm{NaB}$ establish a permissive chromatin state that allows signaling stimulated by subthreshold learning to induce BDNF expression, resulting in robust encoding. The permissive chromatin states induced by exercise and $\mathrm{NaB}$ have epigenetic signatures associated with increased H4K8Ac of promoters specific for $b d n f$ I and $b d n f$ IV (but not $b d n f$ VI), and the respective mRNA transcripts are increased in parallel with the acetylation patterns. Increased $\mathrm{H} 4 \mathrm{~K} 8$ acetylation enhances hippocampal gene expression required to transform a subthreshold OLM training event into long-term memory (previously shown for $\mathrm{NaB}$ treatment; McQuown et al, 2011), indicating that $\mathrm{H} 4 \mathrm{~K} 8$ acetylation may also be an important epigenetic mechanism by which exercise enhances hippocampal function. One mechanism underlying H4K8 acetylation with exercise likely involves enhanced CREB activity (Shen et al, 2001), as exercise increases CREB, and CREB activity in turn increases H4K8 acetylation (Wang et al, 2010). Importantly, activated CREB binds bdnf I and IV promoters (Ou and Gean, 2007) and recruits coactivators with HAT activity required for spatial learning (Alarcon et al, 2004; Korzus et al, 2004; Wood et al, 2005). Another mechanism underlying H4K8 acetylation with exercise may be via decreased HDAC expression by exercise, as has recently been reported (Gomez-Pinilla et al, 2011; Abel and Rissman, 2012). Interestingly, while acetylation at $\mathrm{H} 4 \mathrm{~K} 12$ has been shown to be key for memory consolidation in the aged mouse brain (Peleg et al, 2010), neither exercise nor $\mathrm{NaB}$ significantly increased acetylation of $\mathrm{H} 4 \mathrm{~K} 12$ on $b d n f$ promoters, suggesting that $\mathrm{H} 4 \mathrm{~K} 12$ acetylation is not involved in benefits of exercise and $\mathrm{NaB}$ on subthreshold OLM learning in young adult animals. Although we have focused on $\mathrm{H} 4$ acetylation of BDNF in 
this study, a potential role of $\mathrm{H} 3$ acetylation may also be involved in BDNF induction and the enabling effects of exercise and $\mathrm{NaB}$ on OLM memory, as several learning and memory studies have implicated H3K14 acetylation in the consolidation mechanisms involving gene regulation (Levenson et al, 2004; Chwang et al, 2006; Barrett et al, 2011; Stafford et al, 2012). Ultimately, deciphering the roles of specific acetylation sites is critical for identifying novel therapeutic approaches for cognitive dysfunction, especially considering that histone hypoacetylation is a feature of several neurodegenerative diseases where learning and memory are compromised (Rouaux et al, 2003; Urdinguio et al, 2009).

In summary, these data provide strong support for the concept that exercise engages epigenetic control mechanisms and serves as a natural stimulus that operates in part like $\mathrm{NaB}$ and potentially other HDAC inhibitors. The permissive chromatin state induced by exercise enhances the effect of signaling events, driving gene expression required for long-term memory formation, and enabling learning to occur in conditions that are normally subthreshold for encoding into long-term memory. These findings add to accruing evidence that physical activity is a potent strategy to enhance hippocampal function and cognitive performance.

\section{ACKNOWLEDGEMENTS}

Funding was provided by the following: NIA Grants AG00538 and AG34667 (CWC), T32AG9629 (KAI), NIMH Grant MH081004 (MAW), and NRSA predoctoral fellowship F31DA29368 (MM). We thank Arpine Kirakos for technical assistance with ChIP assays and Dina Matheos for helpful RT-qPCR advice.

\section{DISCLOSURE}

The authors declare no conflict of interest.

\section{REFERENCES}

Abel JL, Rissman EF (2012). Running-induced epigenetic and gene expression changes in the adolescent brain. Int J Dev Neurosci; e-pub ahead of print. doi:pii:S0736-5748(12)00585-0.

Ahlskog JE, Geda YE, Graff-Radford NR, Petersen RC (2011). Physical exercise as a preventive or disease-modifying treatment of dementia and brain aging. Mayo Clin Proc 86: 876-884.

Alarcon JM, Malleret G, Touzani K, Vronskaya S, Ishii S, Kandel ER et al (2004). Chromatin acetylation, memory, and LTP are impaired in CBP + / - mice: a model for the cognitive deficit in Rubinstein-Taybi syndrome and its amelioration. Neuron 42: 947-959.

Barrett RM, Malvaez M, Kramar E, Matheos DP, Arrizon A, Cabrera SM et al (2011). Hippocampal focal knockout of CBP affects specific histone modifications, long-term potentiation, and long-term memory. Neuropsychopharmacology 36: 1545-1556.

Bekinschtein P, Oomen CA, Saksida LM, Bussey TJ (2011). Effects of environmental enrichment and voluntary exercise on neurogenesis, learning and memory, and pattern separation: BDNF as a critical variable? Semin Cell Dev Biol 22: 536-542.

Berchtold NC, Castello N, Cotman CW (2010). Exercise and timedependent benefits to learning and memory. Neuroscience 167: 588-597.
Bredy TW, Wu H, Crego C, Zellhoefer J, Sun YE, Barad M (2007). Histone modifications around individual BDNF gene promoters in prefrontal cortex are associated with extinction of conditioned fear. Learn Mem 14: 268-276.

Calfa G, Chapleau CA, Campbell S, Inoue T, Morse SJ, Lubin FD et al (2012). HDAC activity is required for BDNF to increase quantal neurotransmitter release and dendritic spine density in CA1 pyramidal neurons. Hippocampus 22: 1493-1500.

Cassilhas RC, Lee KS, Fernandes J, Oliveira MG, Tufik S, Meeusen $\mathrm{R}$ et al (2012). Spatial memory is improved by aerobic and resistance exercise through divergent molecular mechanisms. Neuroscience 202: 309-317.

Chwang WB, O’Riordan KJ, Levenson JM, Sweatt JD (2006). ERK/ MAPK regulates hippocampal histone phosphorylation following contextual fear conditioning. Learn Mem 13: 322-328.

Cotman CW, Berchtold NC (2002). Exercise: a behavioral intervention to enhance brain health and plasticity. Trends Neurosci 25: 292-298.

Cotman CW, Berchtold NC (2007). Physical activity and the maintenance of cognition: learning from animal models. Alzheimers Dement 3: S30-S37.

Cotman CW, Berchtold NC, Christie LA (2007). Exercise builds brain health: an interplay of central and peripheral factors. Trends Neurosci 30: 467-471.

Farmer J, Zhao X, van Praag H, Wodtke K, Gage FH, Christie BR (2004). Effects of voluntary exercise on synaptic plasticity and gene expression in the dentate gyrus of adult male SpragueDawley rats in vivo. Neuroscience 124: 71-79.

Gomez-Pinilla F, Zhuang Y, Feng J, Ying Z, Fan G (2011). Exercise impacts brain-derived neurotrophic factor plasticity by engaging mechanisms of epigenetic regulation. Eur J Neurosci 33: 383-390.

Gorski JA, Balogh SA, Wehner JM, Jones KR (2003). Learning deficits in forebrain-restricted brain-derived neurotrophic factor mutant mice. Neuroscience 121: 341-354.

Gottmann K, Mittmann T, Lessmann V (2009). BDNF signaling in the formation, maturation and plasticity of glutamatergic and GABAergic synapses. Exp Brain Res 199: 203-234.

Korzus E, Rosenfeld MG, Mayford M (2004). CBP histone acetyltransferase activity is a critical component of memory consolidation. Neuron 42: 961-972.

Kouzarides T (2007). Chromatin modifications and their function. Cell 128: 693-705.

Levenson JM, O’Riordan KJ, Brown KD, Trinh MA, Molfese DL, Sweatt JD (2004). Regulation of histone acetylation during memory formation in the hippocampus. J Biol Chem 279: 40545-40559.

Lu Y, Christian K, Lu B (2008). BDNF: a key regulator for protein synthesis-dependent LTP and long-term memory? Neurobiol Learn Mem 89: 312-323.

Ma YL, Wang HL, Wu HC, Wei CL, Lee EH (1998). Brain-derived neurotrophic factor antisense oligonucleotide impairs memory retention and inhibits long-term potentiation in rats. Neuroscience 82: 957-967.

McQuown SC, Barrett RM, Matheos DP, Post RJ, Rogge GA, Alenghat $\mathrm{T}$ et al (2011). HDAC3 is a critical negative regulator of long-term memory formation. J Neurosci 31: 764-774.

Middleton LE, Barnes DE, Lui LY, Yaffe K (2010). Physical activity over the life course and its association with cognitive performance and impairment in old age. J Am Geriatr Soc 58: $1322-1326$.

Mizuno M, Yamada K, Olariu A, Nawa H, Nabeshima T (2000). Involvement of brain-derived neurotrophic factor in spatial memory formation and maintenance in a radial arm maze test in rats. J Neurosci 201: 7116-7121.

Mu JS, Li WP, Yao ZB, Zhou XF (1999). Deprivation of endogenous brain-derived neurotrophic factor results in impairment of spatial learning and memory in adult rats. Brain research 835 : $259-265$. 
Neeper SA, Gomez PF, Choi J, Cotman CW (1996). Physical activity increases mRNA for brain-derived neurotrophic factor and nerve growth factor in rat brain. Brain Res 726: 49-56.

Oliff HS, Berchtold NC, Isackson P, Cotman CW (1998). Exerciseinduced regulation of brain-derived neurotrophic factor (BDNF) transcripts in the rat hippocampus. Brain Res Mol Brain Res 61: $147-153$.

Ou LC, Gean PW (2007). Transcriptional regulation of brainderived neurotrophic factor in the amygdala during consolidation of fear memory. Mol Pharmacol 72: 350-358.

Peleg S, Sananbenesi F, Zovoilis A, Burkhardt S, Bahari-Javan S, Agis-Balboa RC et al (2010). Altered histone acetylation is associated with age-dependent memory impairment in mice. Science 328: 753-756.

Rex CS, Lin CY, Kramar EA, Chen LY, Gall CM, Lynch G (2007). Brain-derived neurotrophic factor promotes long-term potentiation-related cytoskeletal changes in adult hippocampus. J Neurosci 27: 3017-3029.

Rouaux C, Jokic N, Mbebi C, Boutillier S, Loeffler JP, Boutillier AL (2003). Critical loss of CBP/p300 histone acetylase activity by caspase-6 during neurodegeneration. EMBO J 22: 6537-6549.

Russo-Neustadt AA, Beard RC, Huang YM, Cotman CW (2000). Physical activity and antidepressant treatment potentiate the expression of specific brain-derived neurotrophic factor transcripts in the rat hippocampus. Neuroscience 101: 305-312.

Sahar S, Reddy MA, Wong C, Meng L, Wang M, Natarajan R (2007). Cooperation of SRC-1 and p300 with NF-kappaB and CREB in angiotensin II-induced IL-6 expression in vascular smooth muscle cells. Arterioscler Thromb Vasc Biol 27: $1528-1534$.

Shen H, Tong L, Balazs R, Cotman CW (2001). Physical activity elicits sustained activation of the cyclic AMP response elementbinding protein and mitogen-activated protein kinase in the rat hippocampus. Neuroscience 107: 219-229.
Stafford JM, Raybuck JD, Ryabinin AE, Lattal KM (2012). Increasing histone acetylation in the hippocampus-infralimbic network enhances fear extinction. Biol Psychiatry 72: 25-33.

Stefanko DP, Barrett RM, Ly AR, Reolon GK, Wood MA (2009). Modulation of long-term memory for object recognition via HDAC inhibition. Proc Natl Acad Sci USA 106: 9447-9452.

Urdinguio RG, Sanchez-Mut JV, Esteller M (2009). Epigenetic mechanisms in neurological diseases: genes, syndromes, and therapies. Lancet Neurol 8: 1056-1072.

van Praag H, Christie BR, Sejnowski TJ, Gage FH (1999). Running enhances neurogenesis, learning, and long-term potentiation in mice. Proc Natl Acad Sci USA 96: 13427-13431.

Vaynman S, Ying Z, Gomez-Pinilla F (2004). Hippocampal BDNF mediates the efficacy of exercise on synaptic plasticity and cognition. Eur J Neurosci 20: 2580-2590.

Vecsey CG, Hawk JD, Lattal KM, Stein JM, Fabian SA, Attner MA et al (2007). Histone deacetylase inhibitors enhance memory and synaptic plasticity via CREB:CBP-dependent transcriptional activation. J Neurosci 27: 6128-6140.

Voss MW, Nagamatsu LS, Liu-Ambrose T, Kramer AF (2011). Exercise, brain, and cognition across the lifespan. J Appl Physiol 111: $1505-1513$.

Wang D, Xia X, Weiss RE, Refetoff S, Yen PM (2010). Distinct and histone-specific modifications mediate positive versus negative transcriptional regulation of TSHalpha promoter. PLOS ONE 5: e9853.

Wood MA, Kaplan MP, Park A, Blanchard EJ, Oliveira AM, Lombardi TL et al (2005). Transgenic mice expressing a truncated form of CREB-binding protein (CBP) exhibit deficits in hippocampal synaptic plasticity and memory storage. Learn Mem 12: 111-119.

Zeng Y, Tan M, Kohyama J, Sneddon M, Watson JB, Sun YE et al (2011). Epigenetic enhancement of BDNF signaling rescues synaptic plasticity in aging. J Neurosci 31: 17800-17810.

Supplementary Information accompanies the paper on the Neuropsychopharmacology website (http://www.nature.com/npp) 